\title{
Discontinuous space variant sub-wavelength structures for generating radially polarized light in visible region
}

Z. Ghadyani

Zahra.Chadyani@physik.uni-erlangen.de

\section{S. Dmitriev}

\section{N. Lindlein \\ G. Leuchs \\ O. Rusina}

I. Harder

\begin{abstract}
Institute for Optics, Information and Photonics, Friedrich-Alexander-University ErlangenNuremberg,Staudtstr. 7/B2, D-91058 Erlangen, Germany

Institute for Optics, Information and Photonics, Friedrich-Alexander-University ErlangenNuremberg,Staudtstr. 7/B2, D-91058 Erlangen, Germany

Institute for Optics, Information and Photonics, Friedrich-Alexander-University ErlangenNuremberg,Staudtstr. 7/B2, D-91058 Erlangen, Germany

Institute for Optics, Information and Photonics, Friedrich-Alexander-University ErlangenNuremberg,Staudtstr. 7/B2, D-91058 Erlangen, Germany

Max-Planck Institute for the Science of Light,Guenther-Scharowsky-Str. 1/Bau 24, D-91058 Erlangen, Germany

Max-Planck Institute for the Science of Light,Guenther-Scharowsky-Str. 1/Bau 24, D-91058 Erlangen, Germany
\end{abstract}

A discontinuous space variant sub-wavelength dielectric grating is designed and fabricated for generating radially polarized light in visible region $(\lambda=632.8 \mathrm{~nm}$ ). The design is based on sub-wavelength silicon nitride structures introducing a retardation of $\pi / 2$ by form birefringence, with space variant orientation of the optical axis. The pattern is divided into concentric ring segments with constant structural parameters, therefore reducing electron-beam writing time significantly. The design avoids the technological challenges encountered in the generation of a continuous space variant grating while maintaining good quality of the resulting polarization mode. [DOI: http://dx.doi.org/ $10.2971 /$ jeos.2011.11041]

Keywords: nonlinear optics, nonlinear slow-mode waveguides, stimulated Raman scattering, self-phase modulation, Silicon Raman amplifier

\section{INTRODUCTION}

The most common polarization modes used in research are spatially homogeneous states of polarization (SOPs). However, the polarization state can, in general be spatially variant. Radially polarized light is an important case in which the polarization state is locally oriented in radial direction. This mode is known to have a large range of applications such as tight focusing of light, optical trapping, laser machining, plasmon excitation, low-loss propagation in metal-clad fibers and the study of nano-structures [1]-[6]. It can be obtained by various methods like interference of two linearly polarized modes [7] or space-variant twisted nematic liquid crystal devices (LCDs) [8]. A common approach to obtain radially polarized light is the usage of space variant waveplates [9]. Due to the limited number of waveplate segments, an additional mode filtering element (Fabry Perot Interferometer) is necessary.

Alternatively, this polarization mode can be made by space variant sub-wavelength structures produced by nanostructuring methods. One possibility is using sub-wavelength metal structures as a radial polarizer [10]. Another possibility is applying form-birefringent elements implemented with dielectric sub-wavelength surface relief gratings analogous to the space variant waveplate [11]. Here the orientation of the optical axis can be changed continuously. The structure can be based either on a space variant quarter waveplate (SVQW) [11] or a space variant half waveplate (SVHW) [12]. Depending on whether the design is based on a quarter or a half waveplate, the structural parameters of the local gratings should be such that the phase retardation is everywhere $\pi / 2$ or $\pi$. In both continuous designs the global area of the elements are limited to $r_{\text {out }} / r_{\text {in }}=d_{\text {max }} / d_{\text {min }}$ for the SVQW and $\sqrt{r_{\text {out }} / r_{\text {in }}}=d_{\text {max }} / d_{\text {min }}$ for the SVHW where $r_{\text {out }}\left(r_{\text {in }}\right)$ is the outer (inner) radius, $d_{\text {max }}$ is the maximum period imposed by Wood's anomaly[13], and $d_{\min }$ is the minimum period that can be fabricated. For instance, the maximum period given by Wood's anomaly for silicon nitride with refractive index of 2.01 for wavelength $632.8 \mathrm{~nm}$ is around $300 \mathrm{~nm}$. We assume the limiting period imposed by the fabrication process is $200 \mathrm{~nm}$. By designing the outer radius to be $1 \mathrm{~mm}$, the inner radius in any approach is $0.66 \mathrm{~mm}$ and $0.44 \mathrm{~mm}$ respectively. With the SVHW a slightly larger area can be achieved, at the cost of more difficult fabrication conditions of the half waveplate, i.e. a higher aspect ratio. Both designs require a continuous variation of the period. On the other hand the retardation is highly dependent on period. Therefore the duty cycle should be adapted for each period within the pattern to obtain the desired retardation. This becomes even more complicated by assuming that in practice the etching depth depends also on 
period and duty cycle.

The continuous designs by Bomzon et al. [11] or by Levy et al. [12] have successfully worked in mid IR spectral region. Applying these designs to shorter wavelengths approach the limitations of current fabrication methods. An example is given in the appendix. Alternatively, it is possible to overcome this problem by a discontinuous design based on quantizing the above mentioned continuous pattern. This principle is applied for the SVHW for the wavelength of $1064 \mathrm{~nm}$ by assuming constant structural parameters and constant orientation of the grating in each segment [14]. The orientation of the grating is changing gradually in adjacent segments. However the SVHW has the disadvantage over the SVQW that it requires higher aspect ratio. In this paper we present a discontinuous design applied to the SVQW by dividing it into concentric ring segments. It is designed to generate radially polarized light in visible region $(\lambda=632.8 \mathrm{~nm})$. The proposed design has three main advantages. First, the symmetry of the design and nearly constant structural parameters leads to more homogeneous etching conditions. Second, the design benefits from low number of data points that reduces the e-beam lithography writing time significantly. Third, a larger active area can be achieved by concentric repetition of the ring segments with increasing inner radius.

In Section 2 the concept of dividing the quarter waveplate design into concentric ring segments is described. The design of the structural parameters is discussed in Section 3. The fabrication and the results are presented in Section 4 . The characterization method and the results for the fabricated element are provided in Section 5. In Section 6 the element is used to generate radially polarized light and the propagating mode is characterized. Finally the paper is concluded in Section 7.

\section{Concentric ring segmentation}

It is known that a grating with sub-wavelength period induces a phase retardation between TE and TM polarization [15]. The structural parameters can be optimized to achieve phase retardation of $\pi / 2$ or $\pi$ to design a quarter waveplate or half waveplate with optical axis parallel to the grating lines. The quarter waveplate requires less height and is therefore less challenging for fabrication. It can produce linearly polarized light if it is illuminated by circularly polarized light. The orientation of linear polarization can be rotated to radial direction by a space varying orientation of the grating vector:

$$
K_{g}=K_{0}(r)\left(\cos \left(\frac{\pi}{4}\right) \hat{r}+\sin \left(\frac{\pi}{4}\right) \hat{\theta}\right),
$$

where $K_{0}(r)=2 \pi / d(r)$ is the modulus of the grating vector for a grating of local period $d(r)$. The $\hat{r}$ and $\hat{\theta}$ are the unit vectors in radial and azimuthal directions respectively. This can be investigated using Jones matrix notation of the local quarter waveplates with an optical axis rotated $45^{\circ}$ with respect to radial direction and multiplying it by the Jones vector of cir- cularly polarized light:

$$
\begin{aligned}
& \left(\begin{array}{cc}
\cos \left(\theta+\frac{\pi}{4}\right) & -\sin \left(\theta+\frac{\pi}{4}\right) \\
\sin \left(\theta+\frac{\pi}{4}\right) & \cos \left(\theta+\frac{\pi}{4}\right)
\end{array}\right)\left(\begin{array}{ll}
1 & 0 \\
0 & \mathrm{i}
\end{array}\right) \\
& \times\left(\begin{array}{cc}
\cos \left(\theta+\frac{\pi}{4}\right) & \sin \left(\theta+\frac{\pi}{4}\right) \\
-\sin \left(\theta+\frac{\pi}{4}\right) & \cos \left(\theta+\frac{\pi}{4}\right)
\end{array}\right)\left(\begin{array}{l}
1 \\
\mathrm{i}
\end{array}\right) \ldots \\
& =(1+\mathrm{i}) e^{\mathrm{i} \theta}\left(\begin{array}{c}
\cos \theta \\
\sin \theta
\end{array}\right)
\end{aligned}
$$

where $\theta$ is the azimuthal angle. Therefore, the outgoing light is radially polarized but up to a phase which should be eliminated by additionally passing it through a spiral phase plate with opposite sign. The grating vector in Equation 1 can describe a continuous pattern with $K_{0}=2 \pi r_{0} / d_{0} r$ where $d_{0}$ is the period at $r=r_{0}$ [11]. As discussed in Section this continuous pattern is just valid in a small area due to constraints on the period. Additionally, it will have inhomogeneous retardation for different periods if a constant height and a constant duty cycle are assumed. The period dependence of the phase retardation is two fold. First according to rigorous calculation in Section 3 the birefringence and consequently phase retardation of a sub-wavelength structure is changing by changing the period. Second, the etching rate in fabrication process depend on the period. This becomes even more critical for smaller periods required for sub-wavelength structures in visible region. Both the birefringence specified by rigorous calculation and etching rate in fabrication process decreased by reducing period so they cannot compensate each other.

To simplify the pattern, the continuity assumption is neglected and the pattern is divided to very thin concentric rings. Each ring segment includes straight grating lines that are oriented $45^{\circ}$ with respect to the local radial line at the average radius of the ring (Figure 1). The size of each ring is determined by the tolerances of the period $\left[d_{\text {min }}, d_{\text {max }}\right]$ and direction of the grating lines inside the ring. The tolerance of the period is the range that the period can be changed without significantly changing the phase retardation. The period tolerance was determined by fabricating a test grating array with various periods and linewidths and measuring the resulting phase retardation. With our fabrication process the best results for retardation of $\pi / 2$ is achieved with periods between 290-300nm and a constant linewidth of 150nm. The maximum deviation of the grating lines from the desired direction is assumed to be $1^{\circ}$.

The radially polarized light is known to have a doughnut intensity distribution. Its size can be specified by the size of resulting doughnut beam after the spiral phase plate which is required to eliminate the extra vortex term in Equation 2. The spiral phase plate is illuminated by a fundamental nonexpanded Gaussian beam of a HeNe laser. The resulting outgoing light is the Laguerre-Gauss LG01 mode. The camera is put in $100 \mathrm{~mm}$ away from spiral phase plate which is the position of the sub-wavelength element in our setup. The size of area containing intensity higher than $1 / e$ of the maximum intensity is measured. For the given laser beam this is an annular area with inner and outer radii $300 \mu \mathrm{m}$ and $1000 \mu \mathrm{m}$ 


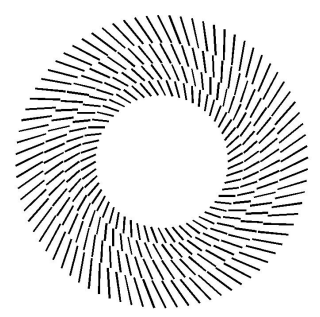

FIG. 1 Concenteric ring segments including straight grating lines rotated $45^{\circ}$ with respect to average radius.

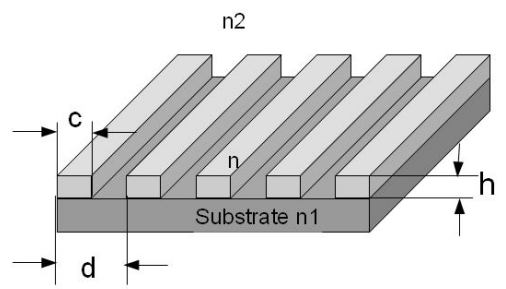

FIG. 2 Geometry and parameters used for structral analysis.

respectively. In order to reduce the e-beam lithography writing time we confine the structure to this annular area. This confined aperture and the segmentation of the pattern lead to diffraction effects which reduce the efficiency of the element.

\section{Analysis of the structural parameters}

Figure 2 illustrates the geometry used for the analysis of the structural parameters in this paper. A periodic binary profile of dielectric grating lines on a substrate is illuminated perpendicularly by a plane wave of wavelength $\lambda=632.8 \mathrm{~nm}$. The design variables are the period $d$, the height (modulation depth) $h$, and the duty cycle or fill factor $c / d$ where $c$ is the width of the grating lines. The refractive index of the first medium (substrate), the surrounding medium and the dielectric lines are denoted by $n_{1}, n_{2}$ and $n$ respectively and all are real values. We denote the amplitude transmission for the TM component by $q_{p}$ and for TE component by $q_{s}$, where $q_{p}=q_{p 0} e^{i \delta_{p}}$ and $q_{s}=q_{s 0} e^{i \delta_{s}}$ are complex values.

The desired properties consist of the transmission ratio between the intensities of the two fundamental polarization components $\eta=\left(q_{p 0} q_{s 0}\right)^{2}$ and the phase retardation defined as $\delta=\delta_{p}-\delta_{s}$. The goal during optimization is $\delta=\pi / 2$, in order to have a quarter waveplate and $\eta=1$, in order to have no additional polarization rotation. The birefringence is a useful descriptive term defined by the difference between the refractive indices for the two orthogonal polarization components $\Delta n=n_{T E}-n_{T M}$. According to the effective medium theory (EMT) [15] which is only approximately correct for very small periods, the birefringence is independent of the grating height and the period. Further investigation by rigorous calculation confirm the height independence

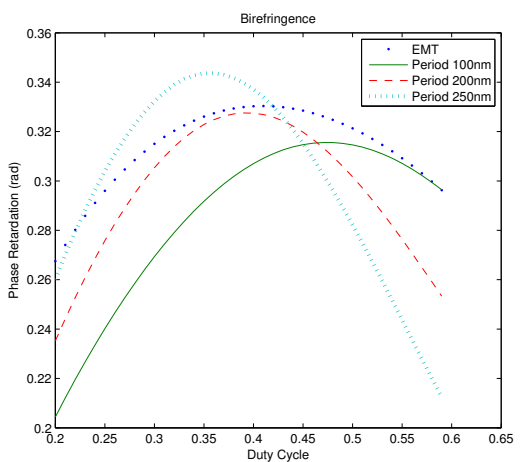

FIC. 3 Birefringence calculated by rigorous calculation for different periods as well as calculated from effective medium theory (EMT) for comparison.

but as shown in Figure 3 the birefringence depends on the period. The birefringence increases with the refractive index $n$ of the material. Therefore silicon nitride is chosen due to its high refractive index of $n(632.8 \mathrm{~nm})=n_{\mathrm{Si}_{3} \mathrm{~N}_{4}}=2.01$ [16], good availability and less challenging structuring process. Fused silica is the substrate medium with refractive index of $n_{1}=n_{\mathrm{SiO}_{2}}=1.457$ in this wavelength region. The medium between the grating lines and above the grating is air with $n_{2}=1$.

Since the limit $d / \lambda \rightarrow 0$ cannot be reached at visible frequencies with conventional grating-fabrication techniques, the effective medium theory cannot be applied for calculating the transmission coefficients with high accuracy. As one of lots of applicable, numerical electrodynamics field solvers we choose the differential method[17]. As can be seen in the Figure 3 the rigorous calculation shows that the birefringence depends not only on the duty cycle and refractive index but also on the period. Therefore, the duty cycle should be varied for quarter waveplate design in a complex pattern like the continuous pattern in the appendix with different periods and constant height. Figure 4 shows the variation of phase retardation and transmission ratio with respect to period and duty cycle for two different height of $460 \mathrm{~nm}$ and $530 \mathrm{~nm}$. The points with the $\pi / 2$ phase retardation are marked by dark red color in Figures $4 \mathrm{a}$ and $4 \mathrm{c}$. Comparing the plots $4 \mathrm{a}$ and $4 \mathrm{c}$ shows a larger range of period is available for higher height and hence higher aspect ratios. The transmission ratio, on the other hand, varies very slowly and is near one where retardation is $\pi / 2$. According to the results for a pattern with varying period, the linewidth can be modified to have a constant retardation. However the assumption of constant height is not valid in fabrication where etching rate is varying for different structural parameters. Therefore, a test element consisting of an array of gratings with different periods and linewidths was fabricated. By measuring the phase retardation, the best structural parameters for a constant retardation was selected for the continuous pattern in the appendix. However in the discontinuous pattern design proposed in Section 2 the period and linewidth are nearly constant. For such a design it is enough to choose one set of structural parameters for the desired retardation of $\pi / 2$. 


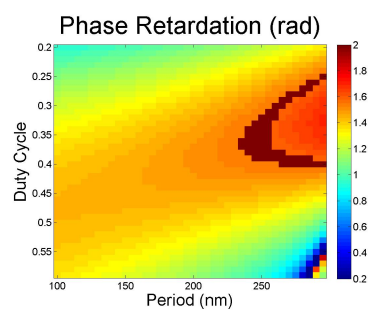

(a)

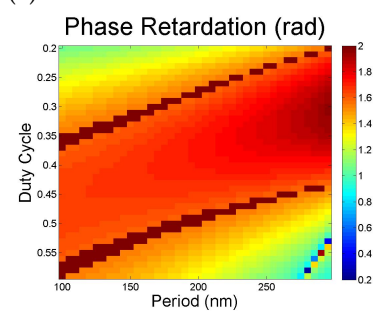

(c)

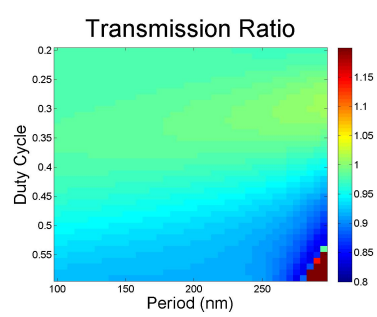

(b)

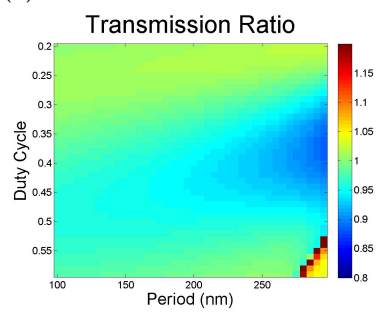

(d)
FIG. 4 Phase retardation and transmission ratio for height $460 \mathrm{~nm}$ ( $\mathrm{a}$ and $\mathrm{b}$ ) and for height 530nm ( $c$ and $d$ ).

\section{Fabrication results}

A silicon nitride layer of 530nm was grown on a fused silica substrate with plasma enhanced chemical vapor deposition (PECVD). A 70nm layer of chromium was evaporated on top of it as hard mask. A 290nm layer of ZEP-520 as resist is spin-coated. The pattern is generated by e-beam lithography (Acceleration voltage: $30 \mathrm{kV}$, Current: $352 \mathrm{pA}$, Dose: $90 \mu \mathrm{C} / \mathrm{cm}^{2}$, Aperture $\left.30 \mu \mathrm{m}\right)$. The generated pattern is transferred to the chromium mask and then to the silicon nitride layer by reactive ion etching. Chlorine and fluorine are used as the main etching gases for chromium and silicon nitride respectively. As mentioned at the end of Section 3, a test element of an array of gratings with different periods and linewidths is fabricated and the retardation of each is measured.

Due to the problems with the continuous patterns (see the appendix), an element according to the design presented in Section 2 was fabricated. The pattern covers an annular area with inner and outer radii $300 \mu \mathrm{m}$ and $1000 \mu \mathrm{m}$ respectively. The writing time for this pattern is 52 hours with our e-beam system that is significantly shorter than the writing time for the continuous design by the factor of $1 / 3$. A small part of the structured area is cut by a focused ion beam (FIB) as shown in figure 5 to check the profile shape and measure the height of the grating. The measurement shows the height of the grating is slightly more than the designed value. The optical characterization by measuring the Stokes parameters also confirms this height deviation. The deviation from the results of the test element is mostly due to the loading effect in the plasma etching process. Therefore, after removing the chromium mask the sample is etched for additional $1 \mathrm{~min}$ to correct the height.

\section{Characterization results}

In general, a full description of a polarizing element can be done by its Mueller matrix. However, the concentric ring seg-

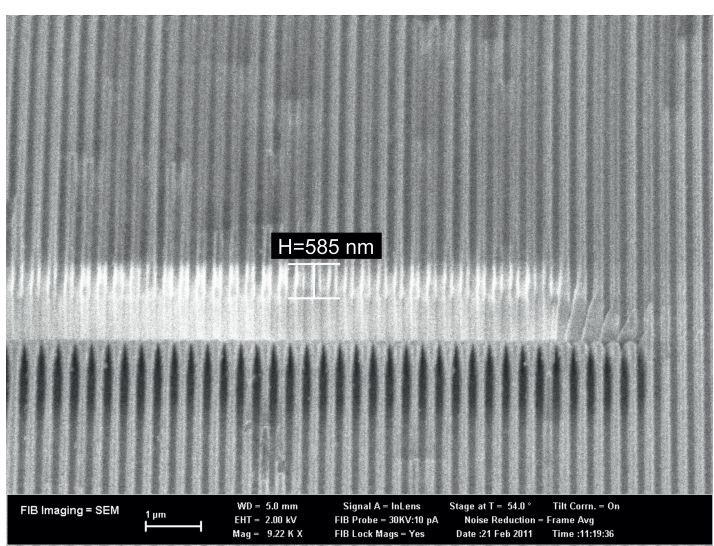

FIG. 5 Cross section of the grating inside the structured area.

mented element is designed to be illuminated by circularly polarized light to generate radially polarized light. Thus, for characterizing the efficiency of the fabricated element it is enough to measure the Stokes parameters of the element illuminated by circularly polarized light and imaged onto the camera. Figure 6 illustrates the setup for Stokes parameters measurement with the rotating quarter waveplate method [18]. In this method the optical beam emerging from the test element with unknown Stokes parameters passes through a quarter waveplate followed by a polarizer denoted by analyser in figure 6 . The transmission axis of the polarizer is fixed in $x$ direction and the second quarter waveplate is rotated by an angle $\theta$. Thus the intensity of the optical beam at the detector is:

$$
I(\theta)=\frac{1}{2}[A-B \sin 2 \theta+C \cos 4 \theta+D \sin 4 \theta],
$$

where

$$
\begin{gathered}
A=\left(S_{0}+\frac{S_{1}}{2}\right), \quad B=S_{3}, \\
C=\frac{S_{1}}{2}, \quad D=\frac{S_{2}}{2},
\end{gathered}
$$

and $S_{0}, S_{1}, S_{2}$ and $S_{3}$ are the unknown Stokes parameters.

Equation 3 is a truncated Fourier series with DC, second harmonic and fourth harmonic terms. The minimum number of data points required can be attained by the Nyquist sampling theorem: a continuous signal can be reconstructed from its samples if it is sampled at a rate at least twice its highest frequency component [19]. In this case the highest frequency is the forth harmonic, so at least 8 data points per full rotation of the quarter waveplate are required. In practice, the quarter waveplate is mounted in a rotation stage and the intensity $I_{n}$ is measured at discrete angular positions $\theta_{n}=n 2 \pi / N$ through $N$ steps. In our experiment we used $N=16$. The coefficients A, B, C and D of Equation 3 and consequently the Stokes parameters can be calculated from a Fourier analysis of the measured intensities. In practice, the two parameters namely orientation $(\psi)$ and ellipticity angle $(\chi)$ of the polarization ellipse are more descriptive and can be derived from Stokes parameters as follows:

$$
\tan 2 \psi=\frac{S_{2}}{S_{1}}, \quad \sin 2 \chi=\frac{S_{3}}{S_{0}},
$$

Figure 7 shows the measured orientation and ellipticity angle. The orientation is in the radial direction and the ellipticity 


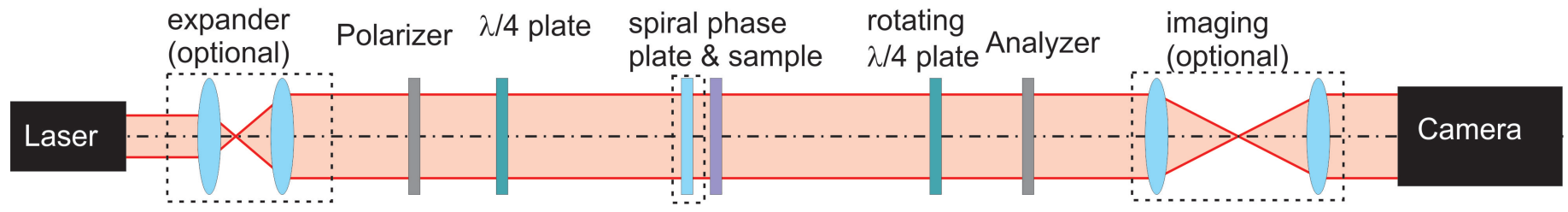

FIG. 6 The setup for measuring the Stokes parameters with optional parts: spiral phase plate for Section 6, beam expander and imaging optics for Section 5 .

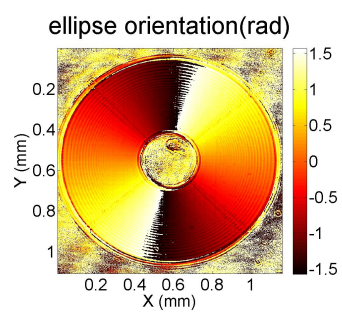

(a)

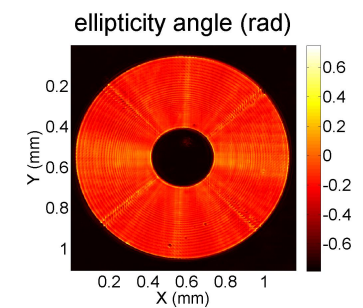

(b)

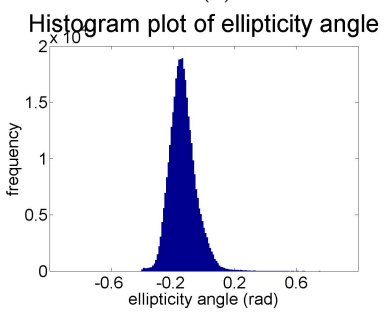

(c)

FIG. 7 Measured parameters of the polarization ellipse.

is normally distributed around 0.136 rad with standard deviation of $0.08 \mathrm{rad}$. The small deviation of the ellipticity angle from zero is due to the fabricated grating height being slightly different from desired calculated height. The results can still be improved by fine tuning the etching process of the element for the correct grating height.

\section{Characterization of the propagating mode}

In the previous section the quality of the fabricated element itself was characterized by measuring the Stokes parameters of the image of the element. In this section the generation and characterization of radially polarized light using the element are investigated. The setup is like the one depicted before in figure 6 but the expanding and imaging parts are removed and the spiral phase plate (SPP) is inserted in front of the concentric ring segmented element. The combination of the element and the SPP is illuminated by a circularly polarized beam with fundamental Gaussian intensity distribution. The beam is further allowed to propagate in free space for one meter. Then the analysing part is inserted right before the camera. In terms of the free space propagation modes, the radially polarized beam of light can be realized as superposition of the two Hermite-Gaussian (HG) beams in two orthogonal bases as follows:

$$
E_{r}=H G_{10} \overrightarrow{e_{x}}+H G_{01} \overrightarrow{e_{y}},
$$

This results in a doughnut shape intensity profile. Figure 8 shows the correspondence of the measured results and the theoretical calculation. The small weak central part is due to diffraction. The polarization ellipse parameters of the prop-

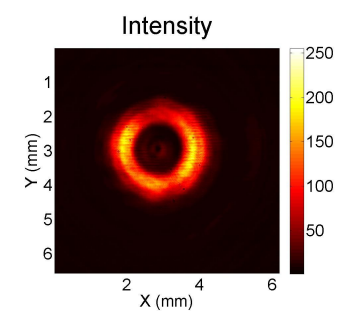

(a)

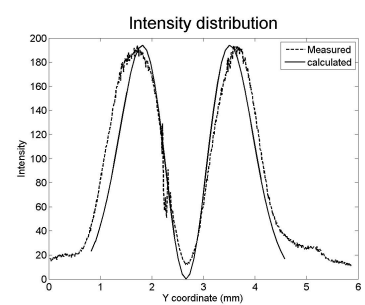

(b)
FIG. 8 Intensity profile for radially polarized light.

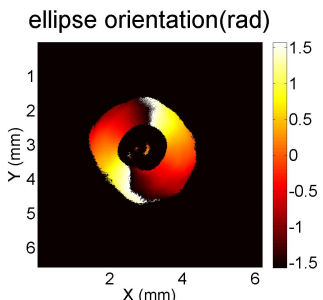

(a)

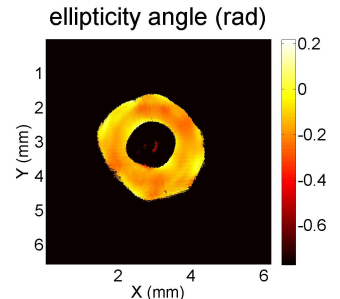

(b)

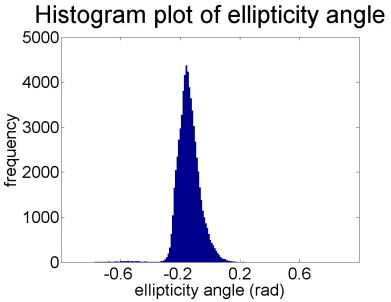

(c)

FIG. 9 The measured parameters of the polarization ellipse of the propagating mode. agating mode are further measured from Stokes parameters (figure 9), using the rotating quarter waveplate method explained in the previous section. The orientation angle of the polarization ellipse is in radial direction as expected. The ellipticity angle is near to zero with normal distribution around $0.14 \mathrm{rad}$ and a standard deviation of $0.07 \mathrm{rad}$. The deviation of the ellipticity from zero is again due to the different height of the grating from the designed value.

\section{Conclusion}

We designed and fabricated a space variant dielectric subwavelength structure to generate radially polarized light at the wavelength $632.8 \mathrm{~nm}$. The generated radially polarized light is characterized by measuring its Stokes parameters and is in good agreement with theory. This shows that the diffraction effects are negligible because of the limited size of the element and segmentation. The results can still be improved by fine tuning the etching process of the element for the correct grating height.The presented design has the advantage that 
there is no principal limit for the size of the structure. Furthermore the simplicity of the design reduces the e-beam writing time by a factor of $1 / 3$. A homogeneous structure is achieved in the etching process due to the fact that the design is symmetric and utilizes nearly constant structural parameters.

\section{Appendix}

The first aim was to fabricate the continuous pattern given in paper [11] where the period and hence the duty cycle are varying. The main problem was a limitation of the e-beam lithography software. The continuous design consists of curved lines with different linewidths for different periods which are best described by polygon area with a high number of data points in a GDSII data file. The e-beam lithography machine stalled during writing this data file.

In the second attempt the aforesaid continuous pattern was fabricated but with constant linewidth that is a mean value of the changing linewidth in the last design. Since this design consists simply of curved lines with constant linewidth, the structure can be described by curved paths which reduces the number of data points in GDSII data file by a factor of 2. As mentioned in Section this continuous pattern covers only a small annular area. Accepting a small discontinuity, we covered the annular area with inner and outer radii $300 \mu \mathrm{m}$ and $1000 \mu \mathrm{m}$ by concentric repetition of the annular area (figure 11). This design was successfully written but took one week to write. The period changed over a shorter interval [250-300nm] and the linewidth remains constant 150nm. The expected retardation variation is 0.14rad according to the rigorous calculation for this variation of structural parameters as shown in figure 10. The fabricated element is characterized with the method described in Section 5. The measured ellipticity angle of the polarization ellipse of the sample illuminated by circularly polarized light is shown in figure 12. The missing part in middle of the sample is just a failure during e-beam lithography writing. It is seen that the measured ellipticity is inhomogeneous and strongly changing for different periods. The retardation variation according to this measurement is 1rad that is significantly different from the expected value. This deviation is due to different etching rates for different periods, also confirmed by measuring the height of the grating lines. Since we do not have the freedom of changing the linewidth in the design the fine tuning of the etching process is not possible. Therefore, the design from Section 2 was developed to overcome this problem.

\section{References}

[1] S. Quabis, R. Dorn, M. Eberler, O. Gloeckl, and G. Leuchs, "Focusing light to a tighter spot", 0pt. Commun. 179, 1455-1461 (2000).

[2] R. Dorn, S. Quabis, and G. Leuchs, "Sharper Focus for a Radially Polarized Light Beam", Phys. Rev. Lett. 91, 233901 (2003).

[3] Q. Zhan, "Trapping metallic Rayleigh particles with radial polarization", Opt. Express 12, 3377-3382 (2004).

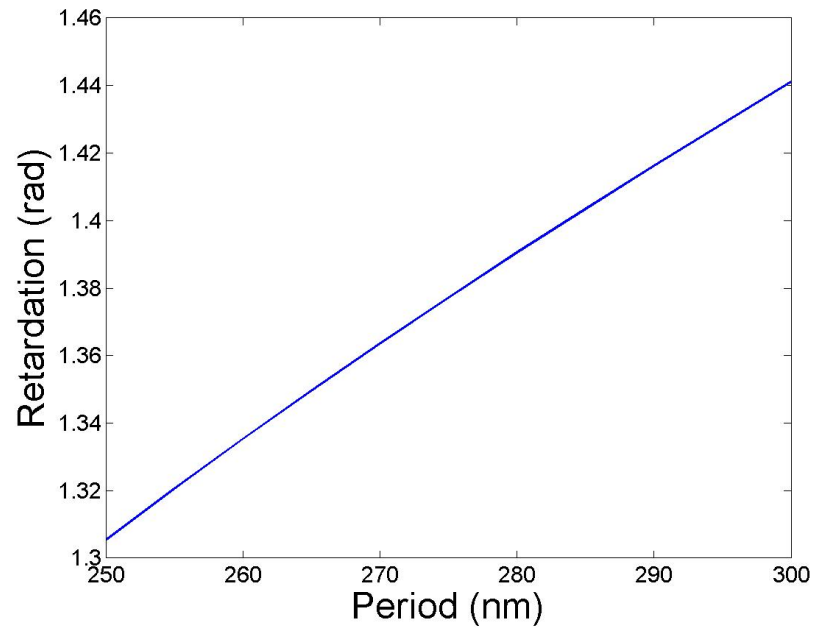

FIG. 10 Calculated phase retardation by varying the period with constant linewidth (150nm) and constant height (460nm).

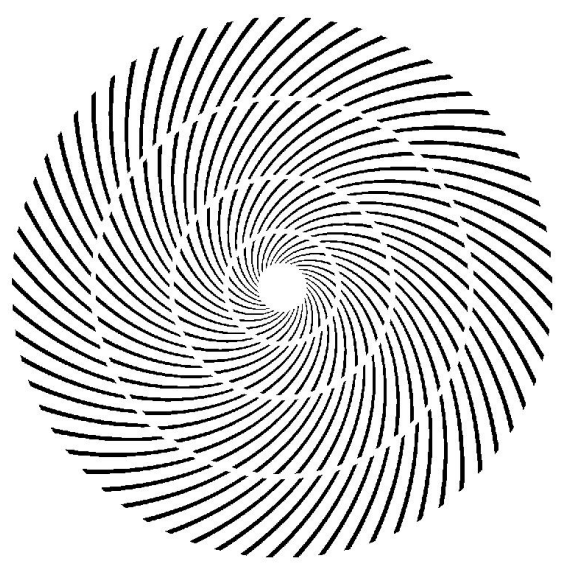

FIG. 11 Schematic picture of the continuous pattern design in the appendix.

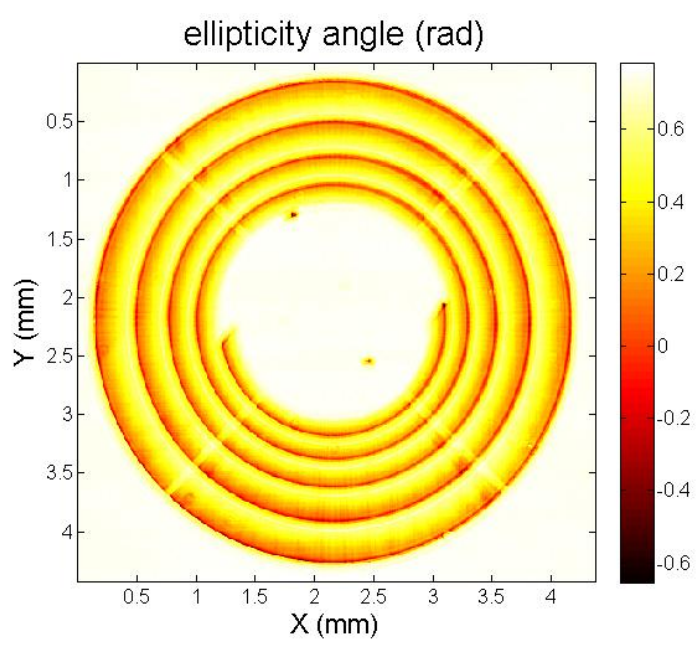

FIG. 12 The ellipticity measured by the method presented in Section 5 for the continuous pattern design sample. 
[4] V. G. Niziev and V. Nesterov, "Influence of beam polarization on laser cutting efficiency", J. Phys. D. Appl. Phys.32, 1455-1461 (1999).

[5] H. Raether, Surface Plasmons on Smooth and Rough Surfaces and Gratings (Spinger-Verlag, 1988).

[6] P. Banzer, U. Peschel, S. Quabis, and G. Leuchs, "On the experimental investigation of the electric and magnetic response of a single nano-structure", 0pt. Express 18, 10905-10923 (2010).

[7] S. C. Tidwell, D. H. Ford, and W. D. Kimura, "Generating radially polarized beams interferometrically", Appl. 0pt. 29, 2234-2239 (1990).

[8] R. Yamaguchi, T. Nose, and S. Sato, "Liquid Crystal Polarizers with Axially Symmetrical Properties", Jpn. J. Appl. Phys. 28, 1730-+ (1989).

[9] S. Quabis, R. Dorn, and G. Leuchs, "Generation of a radially polarized doughnut mode of high quality", Appl. Phys. B - Lasers 0. 81, 597-600 (2005).

[10] Z. Ghadyani, I. Vartiainen, I. Harder, W. Iff, A. Berger, N. Lindlein, and $M$. Kuittinen, "Concentric ring metal grating for generating radially polarized light", Appl. Opt. 50, 2451-2457 (2011).

[11] Z. Bomzon, G. Biener, V. Kleiner, and E. Hasman, "Radially and azimuthally polarized beams generated by space-variant dielectric subwavelength gratings", Opt. Lett. 27, (2002).

[12] U. Levy, C. Tsai, L. Pang, and Y. Fainman, "Engineering spacevariant inhomogeneous media for polarization control", Opt. Lett. 29, 1718-1720 (2004).

[13] R. W. Wood, "On a remarkable case of uneven distribution of light in a diffraction grating spectrum", Phil. Mag. 4, 396-402 (1902).

[14] G. M. Lerman and U. Levy, "Generation of radially polarized light beam using space-variant subwavelength gratings at $1064 \mathrm{~nm}$ ", Opt. Lett. 33, 2782-2784 (2008).

[15] D. C. Flanders, "Submicrometer periodicity gratings as artificial anisotropic dielectrics", Appl. Phys. Lett. 6, 492-494 (1983).

[16] T. Baak, "Silicon oxynitride; a material for GRIN optics", Appl. Optics 21, 1069-1072 (1982).

[17] M. Neviere, Light propagation in periodic media (Marcel Dekker, 2003).

[18] D. H. Goldstein, Polarized Light (Marcel Dekker, 2003).

[19] E. A. Lee and D. G. Messerschmidt (eds.), Digital Communication, 2nd edn. (Kluwer Academic, Boston, MA, 1994). 